\title{
Double-Diffusive Natural Convective Flow of a Nanofluid past an Inclined Wavy Plate in a Non-Darcy Porous Medium
}

\author{
Chukka Venkata Rao \\ Department of Mathematics, \\ M.V.G.R. College of Engineering (A), Vizianagaram-535005, India \\ Corresponding author: venki003@gmail.com \\ Chitteti Ramreddy \\ Department of Mathematics, \\ National Institute of Technology, Warangal-506004, India
}

(Received December 8, 2018; Accepted August 19, 2019)

\begin{abstract}
In this paper, the double-diffusive convective flow along an inclined semi-infinite wavy plate in a nanofluid saturated non-Darcy porous medium is investigated numerically. Following Prandtl's transposition theorem, a coordinate transformation is used to transform the irregular wavy surface into a smooth surface. The convective type thermal boundary condition is taken into account and also the Brownian motion and thermophoresis effects are considered into the present nanofluid model. The governing transport equations are initially reshaped into a system of coupled ordinary differential equations by choosing suitable similarity transformations and then solved numerically by using the Spectral Local Linearization Method (SLLM). The effects of various flow influenced parameters on the fluid flow, heat and mass transfer characteristics are explored and exhibited graphically.
\end{abstract}

Keywords- Double-diffusivity, Inclined wavy plate, Nanofluid, Spectral collocation method.

\section{Introduction}

The double-diffusion is one of the interesting topics in fluid dynamics and it arises in oceans where the convection process is generated by two different density gradients. The doublediffusive natural convection in a Darcy and non-Darcy porous medium has been investigated by Nithiarasu et al. (1997). Khanafer and Vafai (2002) discussed the double-diffusive mixed convection flow in a non-Darcy porous medium. An exhaustive report on the heat and mass transfer due to various fluid flows in a porous medium (both Darcy and non-Darcy models) has been presented by Nield and Bejan (2013).

Most of the authors have analyzed the convective flows with heat transfer over various geometries by assuming the fluid as Newtonian. But, nowadays, the problem of convective flows with heat transfer of non-Newtonian fluids has been one of the active areas in the computational fluid dynamics and one of such fluids is Nanofluid. A suspension of nano-sized fibres or solid particles in conventional fluids (ethylene glycol, water, oil, etc.) is called nanofluid and it is recommended by Choi and Eastman (1995). Both the experimental and theoretical studies have been carried out by several researchers to get enhanced heat transfer rate and higher energy efficiency in various thermal exchange systems for numerous industrial applications. Buongiorno (2006) explored seven slip mechanisms such as fluid drainage, thermophoresis, inertia, Magnus effect, Brownian diffusion, diffusiophoresis, and gravity settling. With this experimentation, he found that the Brownian diffusion and thermophoresis are more significant effects to investigate 
International Journal of Mathematical, Engineering and Management Sciences

Vol. 4, No. 6, 1373-1383, 2019

https://dx.doi.org/10.33889/IJMEMS.2019.4.6-108

the nanofluid flows. A detailed review of nanofluids and their relevant applications have been presented by Das et al. (2007), Das and Stephen (2009), Kakac and Pramuanjaroenkij (2009).

The study of heat and mass transfer over irregular bodies (wavy or non-uniform or rough surfaces) is essential for various heat transfer applications such as flat plate condensers and collectors in refrigerators. The effect of cross diffusion in natural convection flow of a Newtonian fluid along a vertical wavy plate embedded in a Darcy porous medium has been studied by Lakshmi Narayana and Sibanda (2010) whereas Mahdy and Ahmed (2012) considered the problem of laminar natural convective flow along a wavy plate in a porous medium saturated with nanofluid. Further, the natural convection heat and mass transfer over an inclined wavy plate is generally experienced in various devices like electroplating, solar water heaters, processing of heavy metals, etc. The double diffusive natural convection over an inclined wavy plate embedded in a porous medium has been studied by Cheng (2010) and also he investigated the free convection and heat transfer along a wavy inclined surface in a porous medium (see Cheng (2013)). The numerical solutions to natural and mixed convective flows of a nanofluid over an inclined wavy surface in a Darcy porous medium have been presented by Srinivasacharya and Vijay kumar $(2015,2016)$.

The present numerical study aims to investigate the double-diffusive convection in a nanofluid flow over an inclined wavy plate in a non-Darcian porous medium. Pseudo-spectral collocation method along with local linearization technique is employed to solve the present problem numerically [for more details, see Canuto et al. (2006), Motsa (2013), Motsa and Animasaun (2015)]. The effects of relevant physical parameter on the nanofluid fluid flow, heat and mass transfer characteristics have been discussed and shown graphically.

\section{Mathematical Analysis}

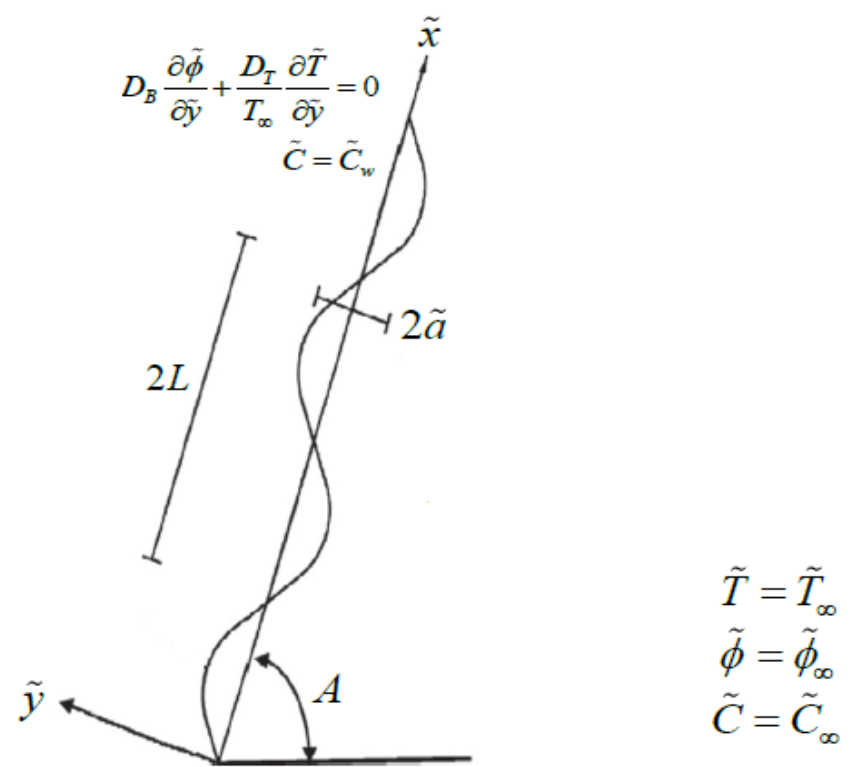

Figure 1. Flow configuration 
International Journal of Mathematical, Engineering and Management Sciences

Vol. 4, No. 6, 1373-1383, 2019

https://dx.doi.org/10.33889/IJMEMS.2019.4.6-108

Consider the laminar, incompressible and 2-D flow over a semi-infinite wavy inclined surface in a nanofluid. The 2-D co-ordinate system is exhibited in Figure 1 . Let $\mathrm{A}\left(0^{\circ} \leq A \leq 90^{\circ}\right)$ be the inclined angle of the wavy surface and the surface of the wavy inclined plate is given by $y_{0}=\sigma(\mathscr{C})=\mathscr{E} \sin (\pi \mathscr{X d L})$ in which $2 L$ and $\mathscr{E}$ are the characteristic length and amplitude of the wavy surface. The wavy surface kept at uniform wall concentration $\mathcal{E}_{w}$ and it assumed to be greater than the ambient concentration $\mathcal{E}_{\infty}^{\infty}$ at any arbitrary reference point in the medium. Further, the ambient temperature and solid volume fraction are considered as $\mathcal{T}_{\infty}$ and $\mathcal{E}_{\infty}$, respectively.

By assuming Oberbeck-Boussinesq approximation and using standard boundary layer approximations, the governing equations can be written as

$$
\begin{aligned}
& \frac{\partial B_{0}}{\partial B_{0}}+\frac{\partial H_{0}}{\partial y_{0}}=0
\end{aligned}
$$

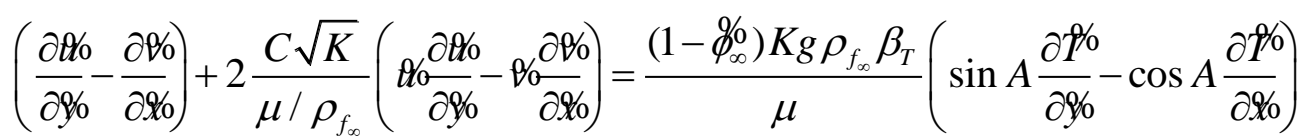

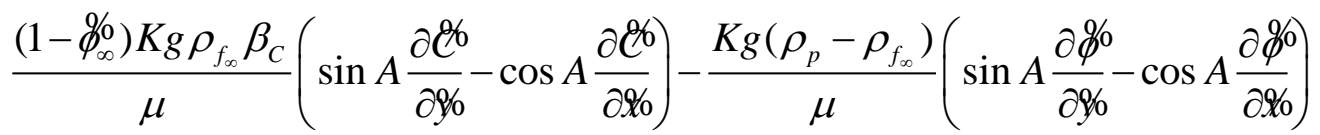

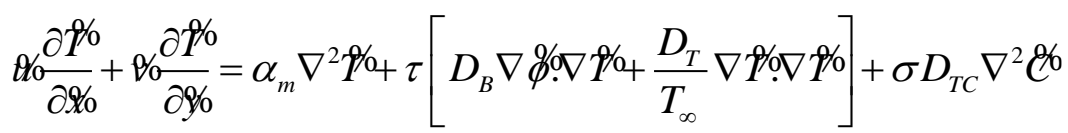

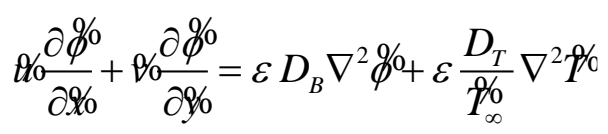

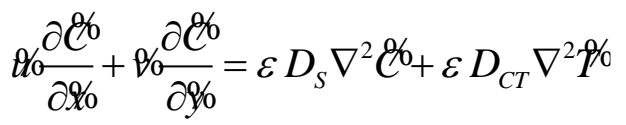

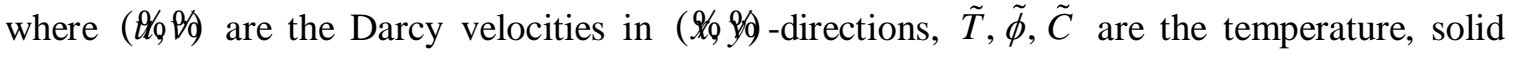
volume fraction, regular concentration, $g$ is the acceleration due to gravity, $K$ is the permeability, $C$ is the Forchheimer's porous inertia constant, $\varepsilon$ is the porosity, $\sigma$ is the electrical conductivity, $\alpha_{m}=k /(\rho c)_{f}$ is the thermal diffusivity, $\tau=\varepsilon(\rho c)_{p} /(\rho c)_{f}$ is the nanofluid heat capacity ratio and $v=\mu / \rho f_{\infty}$ is the kinematic viscosity. Further, $\rho_{f_{\infty}}, \mu, k_{m}, \beta_{T}$ and $\beta_{C}$ are the density of the base fluid, viscosity, thermal conductivity, volumetric thermal and solutal expansion coefficients, while $\rho_{p}$ is the nanoparticles density, $(\rho c)_{p}$ and $(\rho c)_{f}$ are the heat capacity of the nanoparticles and base fluid. Finally, $D_{B}, D_{S}, D_{T C}, D_{T}$ and $D_{C T}$ are the Brownian diffusivity, solutal diffusivity, Dufour type diffusivity, thermophoretic diffusivity, and Soret type diffusivity, respectively. 
International Journal of Mathematical, Engineering and Management Sciences

Vol. 4, No. 6, 1373-1383, 2019

https://dx.doi.org/10.33889/IJMEMS.2019.4.6-108

The related B.C. are

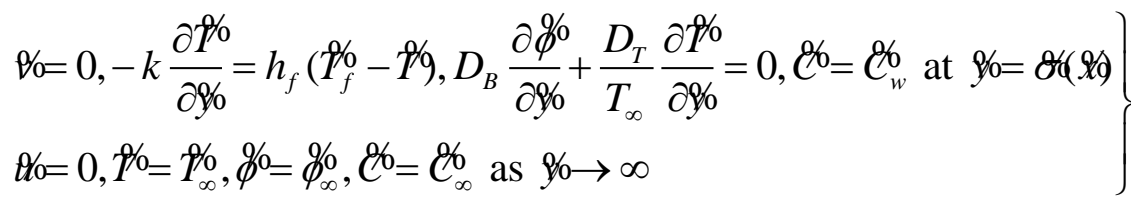

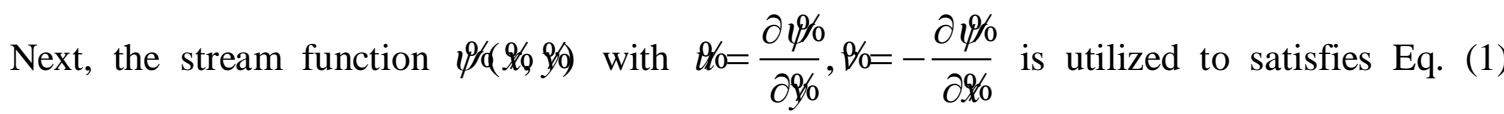
automatically and also we introduce the following dimensionless variables

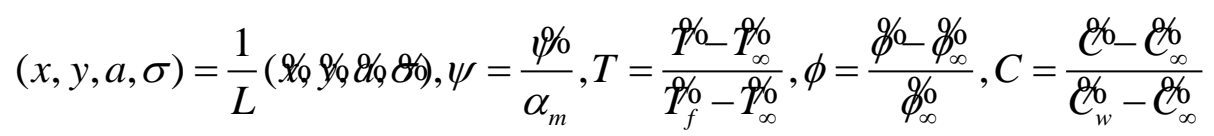

Using Eq. (7), the governing equations (1)-(5) takes the following form

$\frac{\partial^{2} \psi}{\partial x^{2}}+\frac{\partial^{2} \psi}{\partial y^{2}}+\frac{G r^{*}}{R a}\left[2 \frac{\partial \psi}{\partial y} \frac{\partial^{2} \psi}{\partial y^{2}}-2 \frac{\partial \psi}{\partial x} \frac{\partial^{2} \psi}{\partial x^{2}}\right]=R a\left(\sin A \frac{\partial T}{\partial y}-\cos A \frac{\partial T}{\partial x}\right)$

$+R a N c\left(\sin A \frac{\partial C}{\partial y}-\cos A \frac{\partial C}{\partial x}\right)-\operatorname{RaNr}\left(\sin A \frac{\partial \phi}{\partial y}-\cos A \frac{\partial \phi}{\partial x}\right)$

$\frac{\partial \psi}{\partial y} \frac{\partial T}{\partial x}-\frac{\partial \psi}{\partial x} \frac{\partial T}{\partial y}=\nabla^{2} T+N b \nabla \phi . \nabla T+N t \nabla T . \nabla T+N d \nabla^{2} C$

$\frac{\partial \psi}{\partial y} \frac{\partial \phi}{\partial x}-\frac{\partial \psi}{\partial x} \frac{\partial \phi}{\partial y}=\frac{1}{\operatorname{Ln}} \nabla^{2} \phi+\frac{1}{\operatorname{Ln}} \frac{N t}{N b} \nabla^{2} T$

$\frac{\partial \psi}{\partial y} \frac{\partial C}{\partial x}-\frac{\partial \psi}{\partial x} \frac{\partial C}{\partial y}=\frac{1}{L e} \nabla^{2} C+S r \nabla^{2} T$

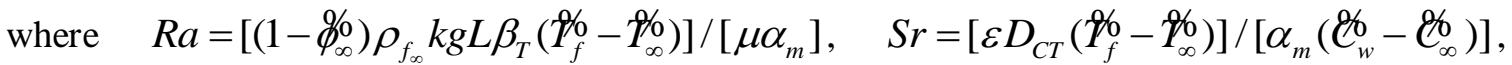

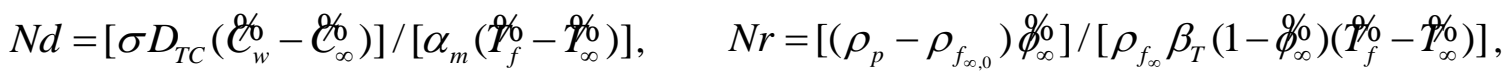

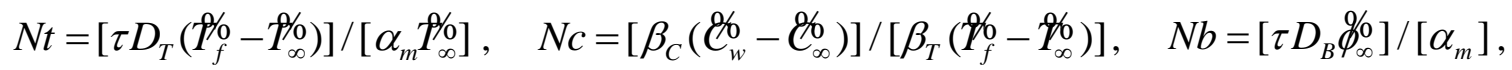
$G r^{*}=\left[C_{f} \sqrt{K} \alpha_{m} R a\right] /[v L], \quad L n=\alpha_{m} / \varepsilon D_{B}$ and $L e=\alpha_{m} / \varepsilon D_{S}$ defines the local Rayleigh number, Soret number, modified Dufour number, nanoparticle buoyancy ratio, thermophoresis parameter, double-diffusive type buoyancy ratio, Brownian motion parameter, non-Darcy parameter, nanoparticle and regular Lewis numbers, respectively.

To transform the irregular surface into a regular surface, we further introduce the co-ordinate transformations as follows

$\bar{x}=x, \bar{y}=R a^{1 / 2}[y-\sigma(x)], \bar{\psi}=R a^{-1 / 2} \psi$ 
International Journal of Mathematical, Engineering and Management Sciences

Vol. 4, No. 6, 1373-1383, 2019

https://dx.doi.org/10.33889/IJMEMS.2019.4.6-108

Using the above transformations (12) and taking $R a \rightarrow \infty$, the equations (8)-(11) takes the following form

$$
\begin{aligned}
& \left(1+\alpha^{2}\right) \frac{\partial^{2} \bar{\psi}}{\partial \bar{y}^{2}}+2 G r^{*}\left(1+a^{2}\right) \frac{\partial \bar{\psi}}{\partial \bar{y}} \frac{\partial^{2} \bar{\psi}}{\partial \bar{y}^{2}}=\left(\frac{\partial \bar{T}}{\partial \bar{y}}+N c \frac{\partial \bar{C}}{\partial \bar{y}}-N r \frac{\partial \bar{\phi}}{\partial \bar{y}}\right)(\sin A+a \operatorname{acos} A) \\
& \left(1+\alpha^{2}\right)\left(\frac{\partial^{2} T}{\partial \bar{y}^{2}}+N b \frac{\partial T}{\partial \bar{y}} \frac{\partial \phi}{\partial \bar{y}}+N t\left(\frac{\partial T}{\partial \bar{y}}\right)^{2}+N d \frac{\partial^{2} C}{\partial \bar{y}^{2}}\right)=\frac{\partial \bar{\psi}}{\partial \bar{y}} \frac{\partial T}{\partial \bar{x}}-\frac{\partial \bar{\psi}}{\partial \bar{x}} \frac{\partial T}{\partial \bar{y}} \\
& \left(1+\alpha^{2}\right) \frac{\partial^{2} \phi}{\partial \bar{y}^{2}}+\left(1+\alpha^{2}\right) \frac{N t}{N b} \frac{\partial^{2} T}{\partial \bar{y}^{2}}=\operatorname{Ln}\left[\frac{\partial \bar{\psi}}{\partial \bar{y}} \frac{\partial \phi}{\partial \bar{x}}-\frac{\partial \bar{\psi}}{\partial \bar{x}} \frac{\partial \phi}{\partial \bar{y}}\right] \\
& \left(1+\alpha^{2}\right) \frac{\partial^{2} C}{\partial \bar{y}^{2}}+\left(1+o^{2}\right) \operatorname{LeSr} \frac{\partial^{2} T}{\partial \bar{y}^{2}}=\operatorname{Le}\left[\frac{\partial \bar{\psi}}{\partial \bar{y}} \frac{\partial C}{\partial \bar{x}}-\frac{\partial \bar{\psi}}{\partial \bar{x}} \frac{\partial C}{\partial \bar{y}}\right]
\end{aligned}
$$

Furthermore, we recommend the following similarity variables to reduce the coupled system of PDEs (13)-(16) into a coupled system of ODEs

$$
\xi=\bar{x}, \eta=\frac{\bar{y}}{\left(1+Q^{2}\right) \xi^{1 / 2}}, \bar{\psi}=\xi^{1 / 2} f(\eta)
$$

Using Eq. (17), we obtain the following equations

$$
\begin{aligned}
& f^{\prime \prime}+2 G r^{*} \frac{(1+Q \ell)}{(1+\alpha)^{2}} f^{\prime} f^{\prime \prime}-\left[T^{\prime}+N c C^{\prime}-N r \phi^{\prime}\right](\sin A+\operatorname{arcos} A)=0 \\
& T^{\prime \prime}+\frac{1}{2} f^{\prime} T^{\prime}+N b T^{\prime} \phi^{\prime}+N t T^{\prime 2}+N d C^{\prime \prime}=0 \\
& \frac{1}{L n} \phi^{\prime \prime}+\frac{1}{2} f \phi^{\prime}+\frac{1}{L n} \frac{N t}{N b} T^{\prime \prime}=0 \\
& \frac{1}{L e} C^{\prime \prime}+\frac{1}{2} f C^{\prime}+S r T^{\prime \prime}=0
\end{aligned}
$$

and the reduced B.C. are

$$
\left.\begin{array}{l}
f=0, T^{\prime}=-B i \xi^{1 / 2}\left(1+\alpha^{2}\right)(1-T), N b \phi^{\prime}+N t T^{\prime}=0, C=1 \text { at } \eta=0 \\
f^{\prime}=0, T=0, \phi=0, C=0 \text { as } \eta \rightarrow \infty
\end{array}\right\}
$$

The non-dimensional local Nusselt number $N u_{9 o}=-\frac{q_{w} \mathscr{Y o}}{k\left(F_{f}^{O}-F_{\infty}^{\circ}\right)}$, nanoparticle Sherwood number

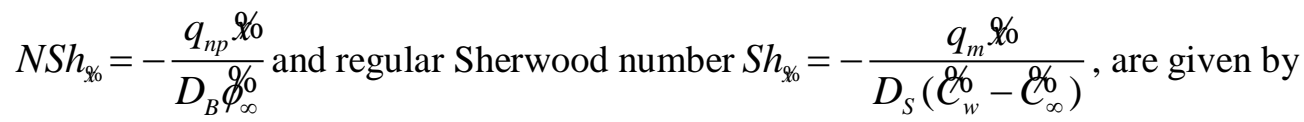


International Journal of Mathematical, Engineering and Management Sciences

Vol. 4, No. 6, 1373-1383, 2019

https://dx.doi.org/10.33889/IJMEMS.2019.4.6-108

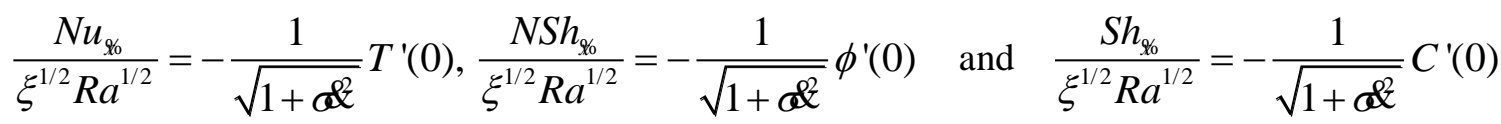

where $q_{w}=-k \hat{n} . \nabla \hat{T}, q_{n p}=-D_{B} \hat{n} . \nabla \mathscr{q}$ and $q_{m}=-D_{S} \hat{n} . \nabla \mathcal{E} c$ are the heat, nanoparticle and

regular mass fluxes at the wavy plate and $\hat{n}=\left(-\infty / \sqrt{1+\alpha^{2}}, 1 / \sqrt{1+a^{2}}\right)$ is the unit outward normal to the wavy surface.

\section{Results and Discussion}

The non-dimensional momentum, energy, solid volume fraction and regular concentration equations (18)-(21) related to the boundary conditions (22) are non-linear and coupled ordinary differential equations for which the analytical solution is out of the scope and thus we solved numerically. In this present work, the governing equations (18)-(21) are solved numerically by using the spectral local linearization method (SLLM) developed by Motsa (2013), Motsa and Animasaun (2015).

The numerical solutions for dimensionless velocity, temperature, solid volume fraction and regular concentration profiles along with the local Nusselt number, local nanoparticle Sherwood number and regular Sherwood number have been evaluated and shown in Figures 2-5. To explore the effects of various physical parameters on the nanofluid flow, heat and mass transfer characteristics, the computations are performed for $N c=0.3, N r=0.3, L n=5.0, L e=5.0, \xi=$ $0.2, N t=0.1, N b=0.1, N d=0.2$ and $S r=0.2$.

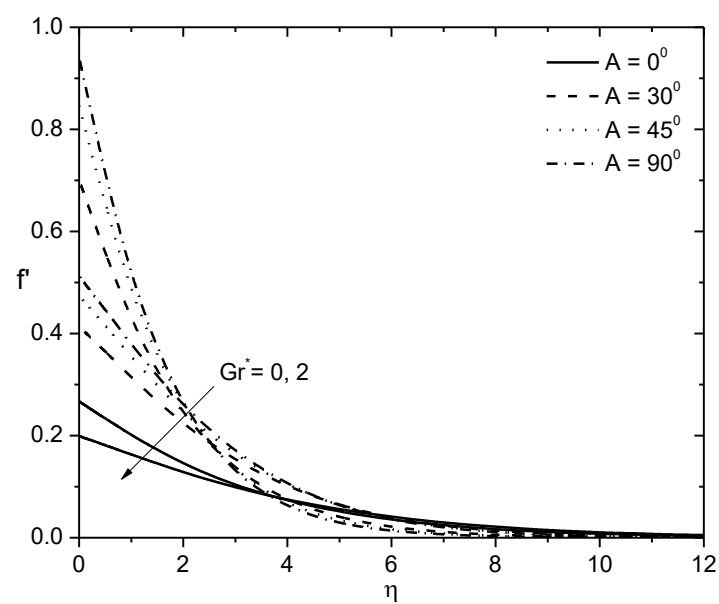

Figure 2. Effects of $G r^{*}$ and $A$ on the velocity

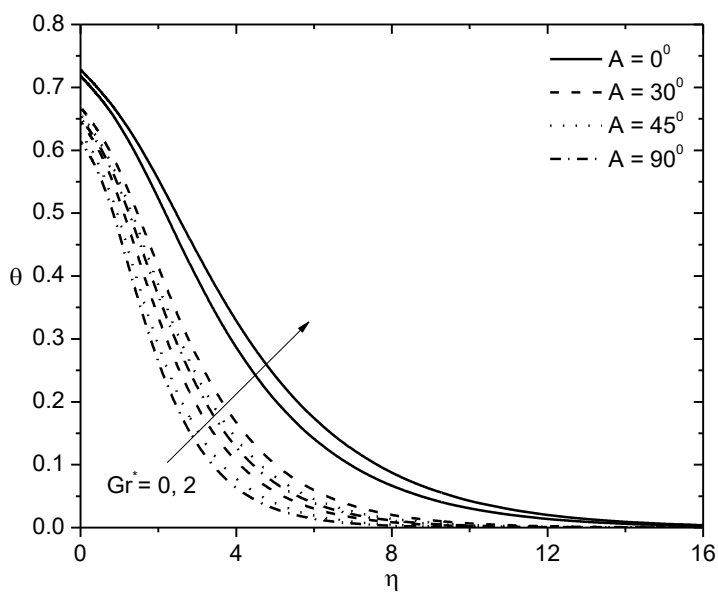

Figure 3. Effects of $G r^{*}$ and $A$ on the Temperature 
International Journal of Mathematical, Engineering and Management Sciences

Vol. 4, No. 6, 1373-1383, 2019

https://dx.doi.org/10.33889/IJMEMS.2019.4.6-108

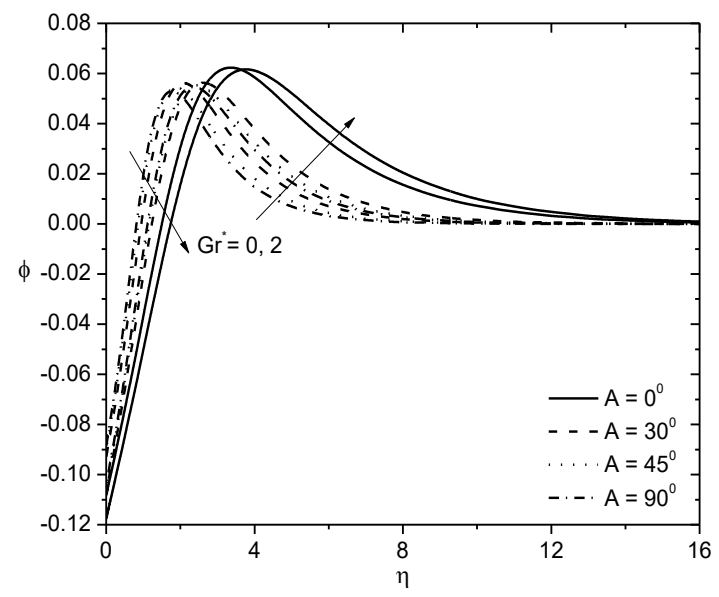

Figure 4. Effects of $G r^{*}$ and $A$ on the solid volume fraction

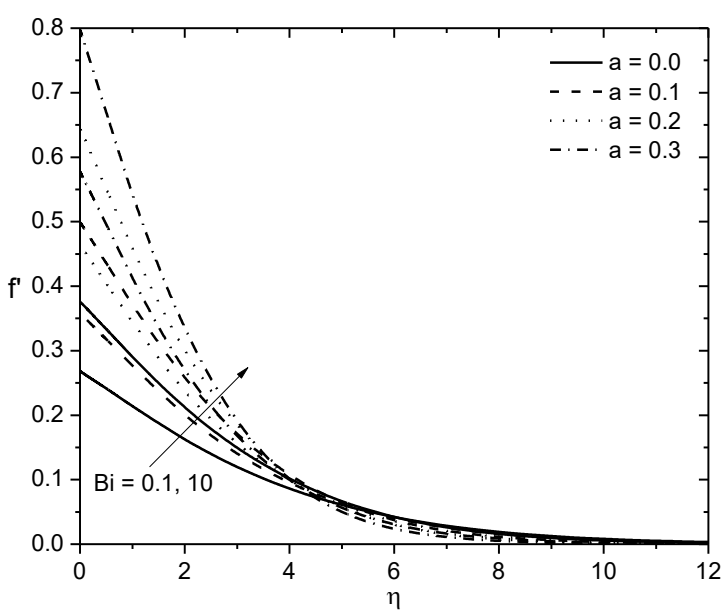

Figure 6. Effects of $B i$ and $a$ on the velocity

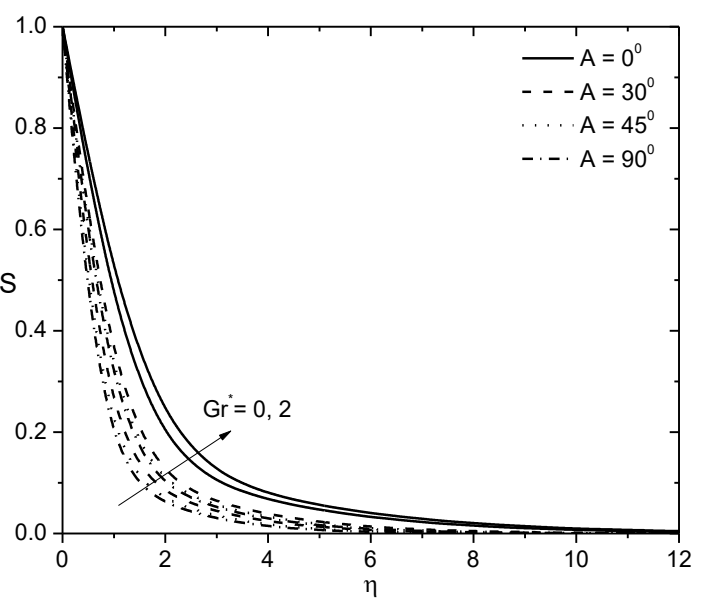

Figure 5. Effects of $G r^{*}$ and $A$ on the regular concentration

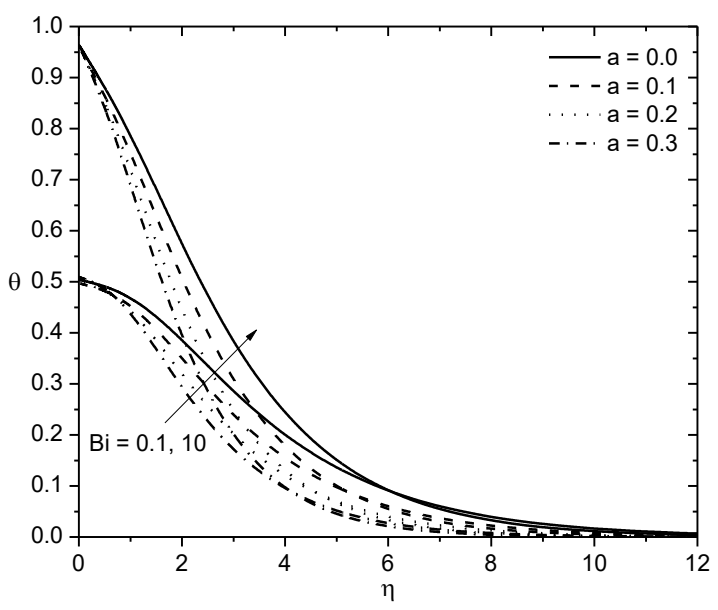

Figure 7. Effects of $B i$ and $a$ on the temperature

Figures 2-5 exhibit the influence of inclined angle $(A)$ and non-Darcy parameter $\left(G r^{*}\right)$ on the nondimensional velocity $\left(f^{\prime}\right)$, temperature $(\theta)$, solid volume fraction $(\phi)$ and regular concentration $(S)$. As limiting cases, the equations for horizontal and vertical surfaces are regained for $A=0^{\circ}$ and $A=90^{\circ}$, respectively. As $A$ increases, the velocity enhances near to the wavy surface but the temperature and regular concentration diminish whereas, the solid volume fraction increases near the surface and decreases far away from the surface as plotted in Figures 2-5. The largest temperature and regular concentration profiles are noticed for horizontal surface case, whereas the smallest values are captured for the vertical surface case. On the other hand, an increase in the non-Darcy parameter leads to decelerate the fluid velocity and increase the temperature in the medium. But, the solid volume fraction profiles decrease near to the surface and increases the regular concentration with increase of $G r^{*}$. 
International Journal of Mathematical, Engineering and Management Sciences

Vol. 4, No. 6, 1373-1383, 2019

https://dx.doi.org/10.33889/IJMEMS.2019.4.6-108

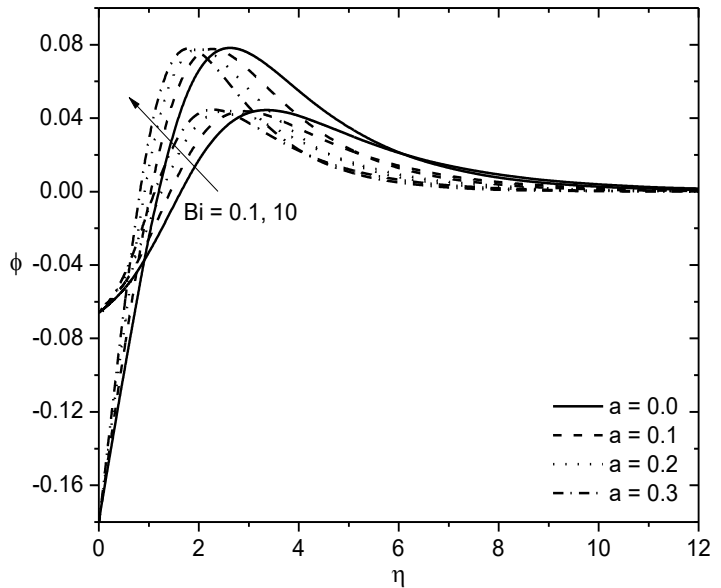

Figure 8. Effects of $B i$ and $a$ on the solid volume fraction

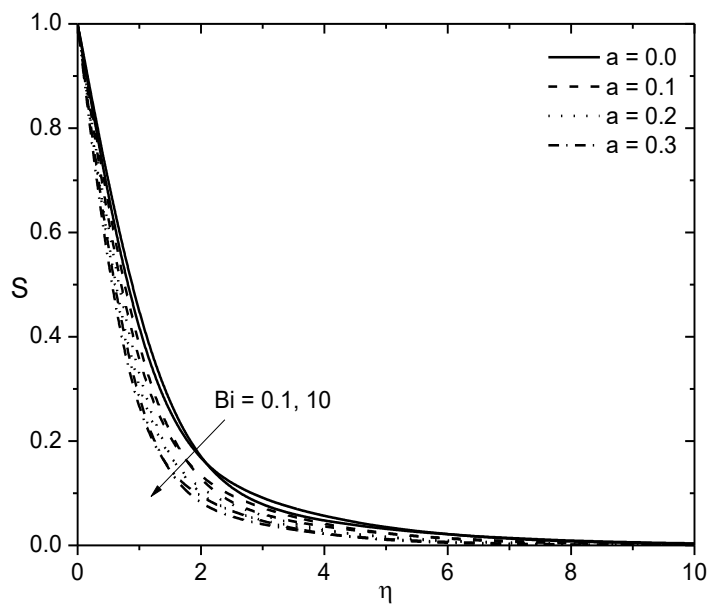

Figure 9. Effects of $B i$ and $a$ on the regular concentration

The impact of Biot number $(B i)$ and wavy amplitude $(a)$ on the non-dimensional velocity $\left(f^{\prime}\right)$, temperature $(\theta)$, solid volume fraction $(\phi)$ and regular concentration $(S)$ are illustrated in Figures 6-9. It is noted that as $a$ increases, the profiles of velocity and volume fraction increase whereas the temperature and regular concentration decreases. Further, an increase in the Biot number causes to accelerate the fluid velocity near to the wavy surface and decelerate the regular concentration as shown in Figures. 6 and 9. The temperature of the fluid enhances whereas the solid volume fraction profile gets increase near to the wavy surface and then decreases far away from the wavy surface.

The influence of non-Darcy parameter $\left(G r^{*}\right)$ and angle of inclination $(A)$ on the streamwise distribution of local Nusselt number $\left(N u_{\mathscr{F}_{0}}\right) /\left(\xi^{1 / 2} R a^{1 / 2}\right)$, local nanoparticle Sherwood number $\left(N S h_{\mathscr{~}}\right) /\left(\xi^{1 / 2} R a^{1 / 2}\right)$ and local regular Sherwood number $\left(S h_{\mathscr{Y}}\right) /\left(\xi^{1 / 2} R a^{1 / 2}\right)$ is shown in Figures 10-

12. These figures reveal that the angle of inclination of the wavy surface enhances the buoyancy force and also assists the fluid flow and then subsequently it leads to an increase in the local Nusselt and regular Sherwood numbers. Whereas a reverse tend is seen in the case of local nanoparticle Sherwood number. As $G r^{*}$ increases, the energy is dissipated as a heat and it increases temperature in the fluid and hence the Nusselt number decreases. Further, the nanoparticle Sherwood number increase and regular Sherwood number decreases with the increasing values of non-Darcy parameter. 
International Journal of Mathematical, Engineering and Management Sciences

Vol. 4, No. 6, 1373-1383, 2019

https://dx.doi.org/10.33889/IJMEMS.2019.4.6-108

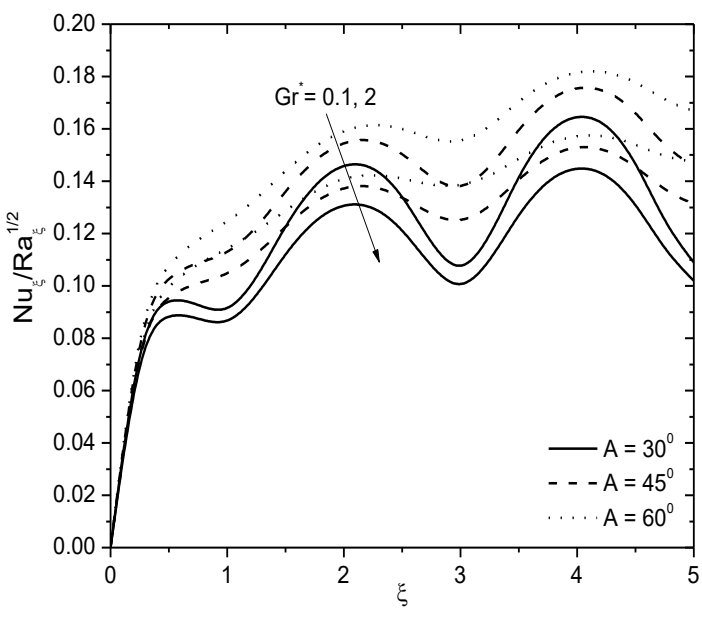

Figure 10. Effects of $G r^{*}$ and $A$ on local Nusselt number

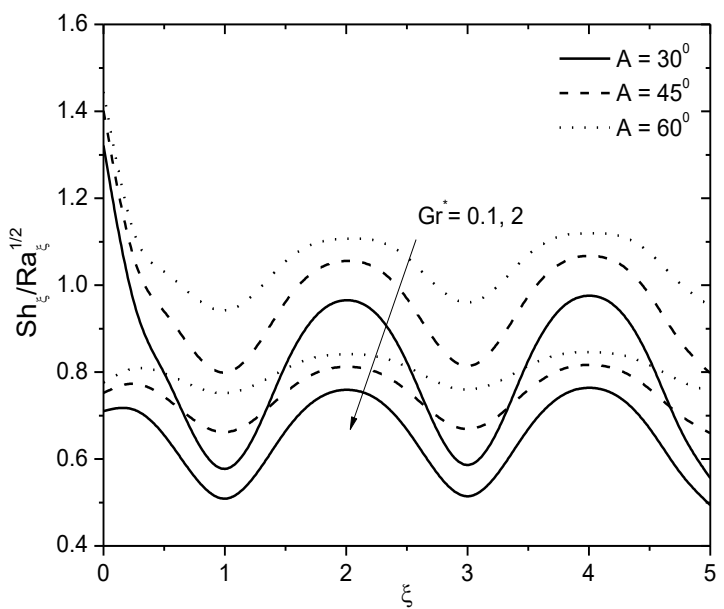

Figure 12. Effects of $G r^{*}$ and $A$ on local regular Sherwood number

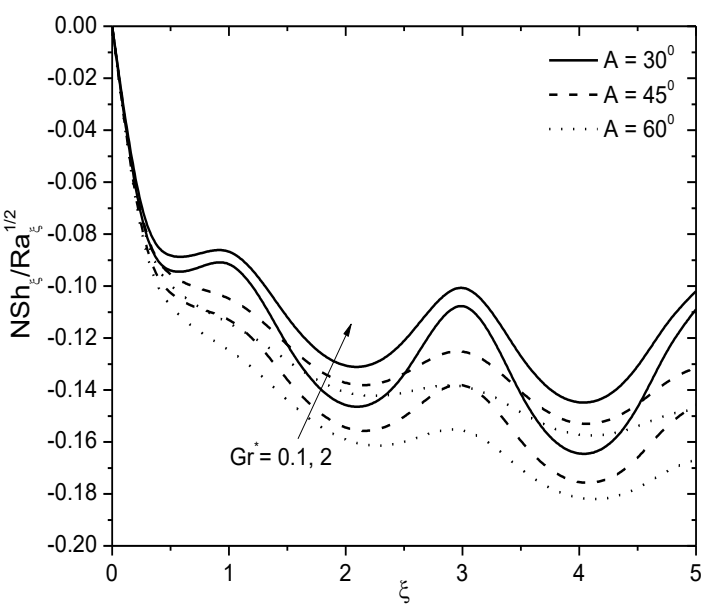

Figure 11. Effects of $G r^{*}$ and $A$ on local nanoparticle Sherwood number

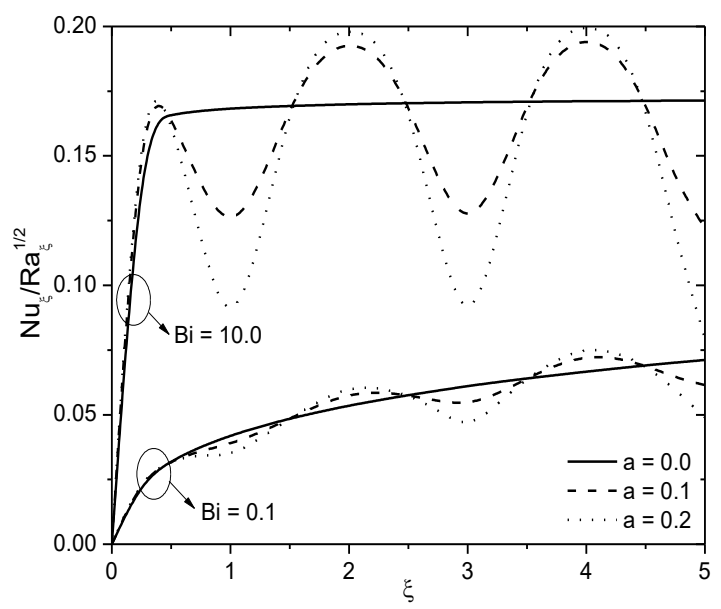

Figure 13. Effects of $B i$ and $a$ on local Nusselt number

Figures 13-15 depict the significance of amplitude of the wavy surface (a) and Biot number $(B i)$ on the streamwise variations of non-dimensional local Nusselt number $\left(N u_{\mathscr{F}_{0}}\right) /\left(\xi^{1 / 2} R a^{1 / 2}\right)$, local nanoparticle Sherwood number $\left(N S h_{\ell \circ}\right) /\left(\xi^{1 / 2} R a^{1 / 2}\right)$ and local regular Sherwood number $\left(S h_{96}\right) /\left(\xi^{1 / 2} R a^{1 / 2}\right)$. These figures show that an increase in the wave amplitude leads more fluctuations in the local Nusselt, local nanoparticle and regular Sherwood numbers. In general, it is clear that the wavy surface turns into more roughened for enhancing values of wave amplitude. Moreover, a remarkable development in the local Nusselt number is noticed for increasing values of Biot number as plotted in Figures 13. But, the local nanoparticle Sherwood number gets decrease whereas the local regular Sherwood number gets increase. 
International Journal of Mathematical, Engineering and Management Sciences

Vol. 4, No. 6, 1373-1383, 2019

https://dx.doi.org/10.33889/IJMEMS.2019.4.6-108

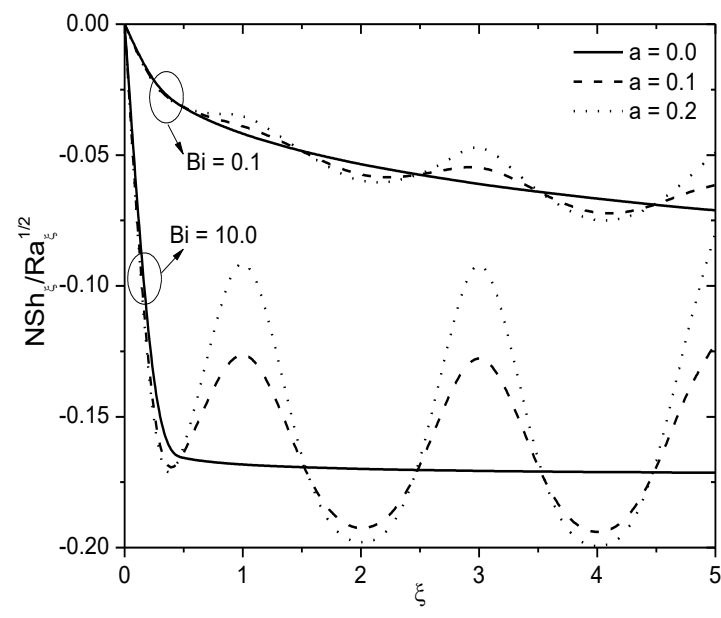

Figure 14. Effects of $B i$ and $a$ on local nanoparticle Sherwood number

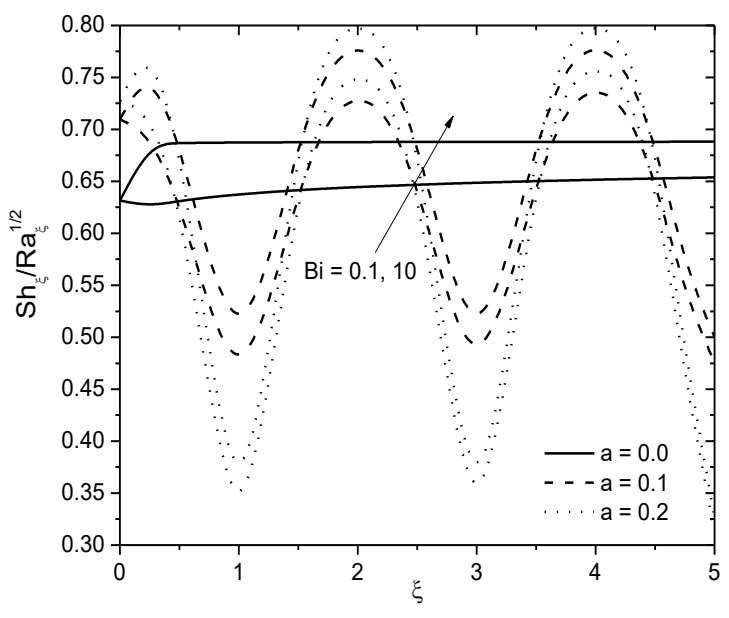

Figure 15. Effects of $B i$ and $a$ on local regular Sherwood number

\section{Conclusions}

In this paper, the double-diffusive type nanofluid flow over an inclined wavy plate in a non-Darcy porous medium is investigated. The spectral local linearization method is employed to obtain the numerical solutions for various values of non-Darcy parameter, Biot number, inclined angle and amplitude. The main outcomes are:

- With an increase in the angle of inclination, the velocity, solid volume fraction, local Nusselt, and regular Sherwood numbers increase and an opposite trend is detected for temperature, regular concentration and local nanoparticle Sherwood number.

- An increase in the wave amplitude enhances the velocity, solid volume fraction, whereas the temperature and regular concentration reduce. Also, the sinusoidal variations are noticed in the Nusselt and Sherwood numbers.

- An increase in the values of $G r^{*}$ significantly reduces the velocity, local heat, and regular mass transfer rate, while the temperature and regular concentration increase.

- As Biot number enhances, the velocity, temperature, volume fraction, local Nusselt and regular Sherwood numbers enhance whereas the local nanoparticle Sherwood number diminishes.

\section{Conflict of Interest}

The authors confirm that this article contents have no conflict of interest.

\section{Acknowledgement}

The authors would like to express their sincere thanks to the referee and for their valuable suggestions towards the improvement of the paper. 
International Journal of Mathematical, Engineering and Management Sciences

Vol. 4, No. 6, 1373-1383, 2019

https://dx.doi.org/10.33889/IJMEMS.2019.4.6-108

\section{References}

Buongiorno, J. (2006). Convective transport in nanofluids. Journal of Heat Transfer, 128(3), 240-250.

Canuto, C., Hussaini, M.Y., Quarteroni, A., \& Zang, T.A. (2006). Spectral methods: fundamentals in single domains. Scientific Computation Series, Springer, Berlin, Heidelberg.

Cheng, C.Y. (2010). Double diffusive natural convection along an inclined wavy surface in a porous medium. International Communications in Heat Mass Transfer, 37(10), 1471-1476.

Cheng, C.Y. (2013). Natural convection heat transfer from an inclined wavy plate in a bidisperse porous medium. International Communications in Heat Mass Transfer, 43, 69-74.

Choi, S.U.S., \& Eastman, J.A. (1995). Enhancing thermal conductivity of fluids with nanoparticles. Argonne National Laboratory, United States.

Das, S.K., \& Stephen, U.S. (2009). A review of heat transfer in nanofluids. Advances in Heat Transfer, 41, 81-197.

Das, S.K., Choi, S. U., Yu, W., \& Pradeep, T. (2007). Nanofluids: science and technology. John Wiley \& Sons, New Jersey.

Kakaç, S., \& Pramuanjaroenkij, A. (2009). Review of convective heat transfer enhancement with nanofluids. International Journal of Heat and Mass Transfer, 52(13-14), 3187-3196.

Khanafer, K., \& Vafai, K. (2002). Double-diffusive mixed convection in a lid-driven enclosure filled with a fluid-saturated porous medium. Numerical Heat Transfer: Part A: Applications, 42(5), 465-486.

Lakshmi Narayana, P.A., \& Sibanda, P. (2010). Soret and Dufour effects on free convection along a vertical wavy surface in a fluid saturated Darcy porous medium. International Journal of Heat and Mass Transfer, 53(15-16), 3030-3034.

Mahdy, A., \& Ahmed, S.E. (2012). Laminar free convection over a vertical wavy surface embedded in a porous medium saturated with a nanofluid. Transport in Porous Media, 91(2), 423-435.

Motsa, S.S. (2013). A new spectral local linearization method for non-linear boundary layer flow problems. Journal of Applied Mathematics, 2013, ID: 423628.

Motsa, S.S., \& Animasaun, I.L. (2015). A new numerical investigation of some thermo-physical properties on unsteady MHD non-Darcian flow past an impulsively started vertical surface. Thermal Sciences, 19(1), 249-258.

Nield, D.A., \& Bejan, A. (2013). Convection in porous media. 4th ed. Springer, New York.

Nithiarasu, P., Seetharamu, K.N., \& Sundararajan, T. (1997). Non-Darcy double-diffusive natural convection in axisymmetric fluid saturated porous cavities. Heat and Mass Transfer, 32(6), 427-433.

Srinivasacharya, D., \& Vijay Kumar, P. (2015). Mixed convection over an inclined wavy surface embedded in a nanofluid saturated porous medium. International Journal of Numerical Methods for Heat and Fluid Flow, 25(8), 1774-1792.

Srinivasacharya, D., \& Vijay Kumar, P. (2016). Mixed convection along an inclined wavy surface in a nanofluid saturated porous medium with wall heat flux. Journal of Nanofluids, 5(1), 120-129. 